\title{
5 VIOLÊNCIA NA ESCOLA E TRANSTORNOS MENTAIS COMUNS EM PROFESSORES 1
}

\author{
| Alyne Fernanda Torres de Lima²; Vanessa Maria da Silva Coêlho³; Albanita Gomes da Costa de Ceballos |
}

\section{RESUMO}

CONTEXTO: A violência que atinge a escola fragiliza os professores, podendo desencadear problemas de saúde física e mental nestes profissionais.

OBJETIVO: Analisar a associação entre a violência na escola e os transtornos mentais comuns (TMC) em professores.

MÉTODOS: Estudo de corte transversal exploratório, do qual participaram 525 professores de Escolas da Rede Pública de um município da Região Metropolitana do Recife (RMR) - Pernambuco. Os dados foram coletados por meio de uma entrevista com os professores que responderam um questionário autoaplicável contendo questões referentes a aspectos sociodemográficos, da atividade profissional, das condições do ambiente de trabalho, violência na escola e saúde. Para avaliação do TMC foi aplicado o Self-Reporting Questionnaire (SRQ-20).

RESULTADOS: Foram investigados 525 professores. A prevalência de TMC foi de 37,1\%. Constatou-se que a agressão física e a agressão verbal contra o professor, a agressão ou ameaça com arma de fogo ou arma branca, o tráfico e o consumo de drogas na escola estão associados à presença de TMC.

CONSIDERAÇÕES FINAIS: Ao mesmo tempo em que se faz necessário investir na promoção da saúde mental e na capacitação do professor para o enfrentamento e a prevenção da violência por meio de medidas de segurança, é preciso intervir no contexto social no qual a escola está inserida. Para tanto, é preciso dar visibilidade ao problema aumentando o debate científico e da sociedade.

\section{PALAVRAS-CHAVE: Transtornos mentais; Violência; Saúde do trabalhador}

\section{RESUMEN}

\section{"Violencia en la escuela y transtornos mentales comunes en pro-} fesores"

CONTEXTO: La violencia que afecta a la escuela debilita a los profesores, pudiendo desencadenar problemas de salud física y mental. OBJETIVO: Analizar la asociación entre la violencia en la escuela y los Trastornos Mentales Comunes (TMC) en los profesores.

METODOLOGIA: Estudio de corte transversal exploratório. Participaron 525 profesores de Escuelas de la Red Pública de un municipio de la Región Metropolitana de Recife (RMR) - Pernambuco. Los datos fueron recolectados a través de una entrevista con los profesores, quienes respondieron un cuestionario autoaplicable que contenía cuestiones referentes a los aspectos sociodemográficos, la actividad profesional, las condiciones del ambiente de trabajo, la violencia en la escuela y la salud. Para la evaluación del TMC se aplicó el SelfReporting Questionnaire (SRQ-20).

RESULTADOS: Se investigaron 525 profesores. La prevalencia de TMC fue del $37,1 \%$. El estudio constató que la agresión física y la agresión verbal contra el profesor, la agresión o amenaza con arma de fuego o arma blanca, el tráfico y el consumo de drogas en la escuela están asociados a la presencia de TMC.

CONSIDERACIONES FINALES: Al mismo tiempo que se hace necesario invertir en la promoción de la salud mental y en la capacitación del profesor para el enfrentamiento y la prevención de la violencia por medio de medidas de seguridad, es necesario intervenir en el contexto social en el cual la escuela está inserta. Para ello, es necesario dar visibilidad al problema aumentando el debate científico y la sociedad.

\section{DESCRIPTORES: Trastornos mentales; Violencia; Salud laboral}

\begin{abstract}
"Violence at school and common mental disorders in teachers"

BACKGROOUND: The violence that reaches the school weakens the teachers and can trigger problems of physical and mental health in these professionals.

AIM: To analyze the association between violence in school and Common Mental Disorders (CMD) in teachers.

METHODS: Exploratory cross-sectional study, in which 525 teachers from Public School Schools participated in a city in the Metropolitan Region of Recife (RMR) - Pernambuco. Were collected through an interview with the teachers, who answered a self-administered questionnaire containing questions regarding sociodemographic aspects, professional activity, working environment conditions, school violence and health. Self-Reporting Questionnaire (SRQ-20) was applied for TMC evaluation.

RESULTS: 525 teachers were investigated. The CMD prevalence was $37,1 \%$. Was found that physical aggression and verbal aggression against the teacher, aggression or threat with a firearm or white weapon, drug trafficking and consumption in school are associated with the presence of CMD.

FINAL CONSIDERATIONS: At the same time that it is necessary to invest in the promotion of mental health and in the training of teachers to confront and prevent violence through security measures, it is necessary to intervene in the social context in which the school is inserted. In order to do so, it is necessary to give visibility to the problem by increasing the scientific and social debate.
\end{abstract}

\section{KEYWORDS: Mental disorders; Violence; Occupational health}

Submetido em 19-06-2017

Aceite em 08-11-2017

\footnotetext{
1 Este artigo é resultado de Dissertação de Mestrado intitulada "A violência na escola e os transtornos mentais comuns (TMC) em professores de escolas municipais de Jaboatão dos Guararapes - Pernambuco", defendida pela primeira autora no Programa de Pós-Graduação em Saúde Coletiva da Universidade Federal de Pernambuco, sob orientação da terceira autora, em março de 2014. As autoras declaram que contaram com recursos financeiros do Edital Universal - CNPq para a elaboração deste trabalho. 2 Biomédica; Mestre em Saúde Coletiva, Rua Edgar Valois, nº 236, 55644-178 Gravatá, PE, Brasil, fernandalyne@hotmail.com

3 Fisioterapeuta; Mestranda na Universidade Federal de Pernambuco, Programa de Pós-Graduação, 50630-620 Recife, PE, Brasil, vanessamscoelho@outlook.com

4 Fonoaudióloga; Doutora em Saúde Pública; Professora na Universidade Federal de Pernambuco, 50740-600, Recife, PE, Brasil, albanita.costa@ufpe.br
}

Citação: Lima, A. F. T., Coêlho, V. M. S., \& Ceballos, A. G. C. (2017). Violência na escola e transtornos mentais comuns em professores. Revista Portuguesa de Enfermagem de Saúde Mental (18), 31-36. doi: 10.19131/rpesm.0189 


\section{INTRODUÇÃO}

A violência é um problema social e histórico que atinge a escola manifestando-se na forma de indisciplina, intolerância e comportamentos agressivos. Atos de depredação do patrimônio, invasões, ameaças, agressões verbais e físicas envolvendo alunos, professores e funcionários em conflitos por delimitação de espaço e poder, levam a discussões sobre a conduta de contenção da violência na escola e sobre a repercussão da mesma na qualidade de vida e na saúde de quem a vivencia (Santos, Vidal, Bittencourt, Boery e Sena, 2011).

Cerca de $80 \%$ dos professores referem já ter vivenciado algum episódio de violência no ambiente escolar, sendo a violência contra o próprio docente a forma mais frequente (Ristum, 2010), encontrando-se este profissional, geralmente, despreparado para atuar frente ao conflito (Oliveira, 2012).

Ser alvo de violência pode provocar danos à saúde dos indivíduos e afetar a integridade física e psíquica dos trabalhadores, causando sintomas de origem psicossomática, desencadeamento ou agravamento de doenças, alterações no sono, depressão, ansiedade e outros (Sousa, 2013). Devido às condições de trabalho diárias, problemas relacionados à saúde mental são frequentemente relatados pelos professores (Ferreira, 2010), sendo este um grande entrave para o bem-estar dos docentes (Oliveira, 2013).

Dentre os problemas de saúde mental, destacam-se os Transtornos Mentais Comuns (TMC) que são caracterizados como sintomas não-psicóticos como insônia, fadiga, irritabilidade, esquecimento, dificuldade de concentração e queixas somáticas (Goldberg e Huxley, 1992).

Estudos realizados no Brasil com professores apontaram uma prevalência de TMC que variou de 17,8\% (Silva, e Silva, 2013) a 54,7\% (Oliveira, 2013). Estes profissionais estão mais propensos ao sofrimento psíquico, sendo sua presença mais comum em docentes vítimas de violência no ambiente escolar e que tiveram algum tipo de conflito com alunos e pais de alunos (Maciel, Nogueira, Maciel, e Aquino, 2012).

Assim, diante da magnitude do problema da violência na escola e da hipótese de que esta violência afete a saúde mental dos professores, este estudo teve como objetivo analisar a associação entre a violência na escola e os transtornos mentais comuns (TMC) em professores das Escolas da Rede Pública de um município na Região Metropolitana do Recife (RMR) - Pernambuco.

\section{MÉTODOS}

A presente pesquisa segue os moldes de um estudo de corte transversal exploratório e faz parte de um projeto estruturante intitulado "Condições de Trabalho e Saúde do Professor". Este foi previamente aprovado pelo Comitê de Ética em Pesquisa do Centro de Ciências da Saúde da Universidade Federal de Pernambuco (CEP/ CCS/UFPE) sob o registro CAAE - 0489.0.172.000-11 e autorizada pela Secretaria de Educação do Município de Jaboatão dos Guararapes.

Jaboatão dos Guararapes está situado na Região Metropolitana do Recife, Pernambuco, em zona urbana e no litoral, distante $20 \mathrm{~km}$ da capital do Estado. É composto por 686.122 habitantes, o Îndice de Desenvolvimento Humano Municipal (IDHM) é de 0,717 e o Índice de Gini de 0,59 (Base de Dados do Estado [BDE], 2016). A rede de educação pública municipal conta com 118 escolas de ensino fundamental e 85 unidades de ensino pré-escolar, 2.228 professores e 48.754 estudantes (Instituto Nacional de Estudos e Pesquisas Educacionais Anísio Teixeira [INEP], 2015).

Para cálculo do tamanho da amostra, foi utilizado o módulo StatCalc do programa EpiInfo versão 3.5.1. Os parâmetros de cálculo foram: estudo de corte transversal, frequência de TMC de $41,5 \%$ em professores do ensino fundamental e médio (Delcor et al., 2004), intervalo de confiança de $95 \%$, poder do estudo de $80 \%$ e razão de chances igual a 2. O resultado foi de amostra mínima composta por 252 professores. Considerando possíveis recusas e perdas ou problemas no preenchimento dos questionários, foi acrescido $40 \%$ ao número de tamanho de amostra e desta forma, a amostra calculada final do estudo foi de 353 professores. Os critérios de seleção foram: ser professor do quadro permanente da secretaria municipal de educação e estar participando das sessões de educação continuada ofertadas pela secretaria de educação. As atividades de educação continuada são oferecidas rotineiramente aos docentes e todos são convidados a participar. Todos os participantes que consentiram em participar da pesquisa assinaram o Termo de Consentimento Livre e Esclarecido (TCLE). Os sujeitos responderam a um questionário autoaplicável contendo questões sociodemográficas, da atividade profissional, das condições do ambiente de trabalho, violência na escola e saúde. A violência na escola foi analisada com referência aos seis meses anteriores ao estudo nas dimensões de agressão ou ameaça física e verbal e consumo ou venda de drogas e álcool. As questões foram desenvolvidas pelos autores da pesquisa. 
Os transtornos mentais comuns foram avaliados utilizando o Self Reporting Questionnaire (SRQ-20) (World Health Organization [WHO], 1994), versão em português. O SRQ-20 foi desenvolvido pela Organização Mundial de Saúde com o objetivo de avaliar os TMC em países em desenvolvimento, sendo comumente utilizado como um instrumento de triagem no Brasil e em outros diversos países. É um instrumento autoaplicável composto por 20 questões. Sobre as características do instrumento, estudos na população brasileira mostram boa consistência interna $(\alpha=0,81)$ (Santos, Araújo e Oliveira, 2009), sensibilidade de $85,0 \%$ e especificidade de 80,0\% (Mari e Williams, 1986).

No presente estudo, para compor a variável TMC foi atribuído 1 ponto para cada resposta positiva e zero ponto para cada resposta negativa. Após o somatório, foi considerado suspeito de TMC (TMC Sim) os pesquisados com pontuação igual ou maior a 8 e não suspeito de TMC (TMC Não) os pesquisados com pontuação menor ou igual a 7, sem distinção de sexo.

Para a digitação dos dados foi usado o programa EPI$\mathrm{INFO}^{\oplus}$ na versão 3.5.2. Para análise dos dados foi utilizado o IBM SPSS Statistics versão 20.0. A descrição das variáveis se deu por meio de análise de frequências e investigou-se a associação da violência com os transtornos mentais comuns, estimando-se os odds ratio (OR) simples e os intervalos de confiança a 95\% (IC95\%). Por se tratar de um estudo de caráter exploratório, não foram analisados fatores de confundimento ou interação.

\section{RESULTADOS}

Devido a grande adesão dos pesquisados, a pesquisa coletou informações de 525 sujeitos. Não foram registradas perdas ou recusas.

Dentre os professores pesquisados, a maioria era do sexo feminino $(86,1 \%)$, tinha idade maior que 36 anos de idade $(62,5 \%)$, cor da pele auto referida não branca $(72,2 \%)$ e heterossexuais $(94,3 \%)$. Houve predomínio de professores especializados e pós-graduados (53,5\%). A renda média do domicílio era menor ou igual a dez salários mínimos $(68,4 \%)$ tendo quatro ou mais dependentes dessa renda $(54,3 \%)$.

Alguns entrevistados já trabalhavam como professores há vinte anos ou mais (26,5\%) e alguns referiram uma carga horária igual ou maior que quarenta horas semanais $(86,5 \%)$. Sobre a valorização profissional, os professores responderam que se sentiam valorizados pelos colegas de trabalho $(93,0 \%)$, pela chefia $(88 \%)$ e pelos alunos $(93,9 \%)$. Sobre a saúde geral dos professores, aproximadamente $70 \%$ dos pesquisados referiu ter faltado ao trabalho por problema de saúde 5 ou mais vezes nos últimos 12 meses. Apenas 8,8\% referiu ser fumante e $13,1 \%$ referiu consumir bebida alcoólica regularmente. A frequência de TMC foi igual a 37,1\% (Tabela 1).

Tabela 1 - Prevalência dos Sintomas Psíquicos Avaliados pelo Self-Reporting Questionnaire (SRQ-20) e de

Transtornos Mentais Comuns (TMC) em Professores da

Rede Municipal de Ensino de Jaboatão dos Guararapes-PE

\begin{tabular}{|c|c|c|}
\hline Variáveis & $\mathrm{N}=525$ & $\%$ \\
\hline \multicolumn{3}{|c|}{ Dores de cabeça frequentes } \\
\hline Não & 300 & 57,1 \\
\hline Sim & 225 & 42,9 \\
\hline \multicolumn{3}{|c|}{ Falta de apetite } \\
\hline Não & 443 & 84,4 \\
\hline Sim & 82 & 15,6 \\
\hline \multicolumn{3}{|c|}{ Dorme mal } \\
\hline Não & 335 & 63,8 \\
\hline Sim & 190 & 36,2 \\
\hline \multicolumn{3}{|c|}{ Assusta-se com facilidade } \\
\hline Não & 360 & 68,6 \\
\hline Sim & 165 & 31,4 \\
\hline \multicolumn{3}{|c|}{ Tem tremores nas mãos } \\
\hline Não & 460 & 87,6 \\
\hline Sim & 65 & 12,4 \\
\hline \multicolumn{3}{|c|}{ Sente-se nervoso, tenso ou preocupado } \\
\hline Não & 294 & 56,0 \\
\hline Sim & 231 & 44,0 \\
\hline \multicolumn{3}{|c|}{ Tem má digestão } \\
\hline Não & 366 & 69,7 \\
\hline Sim & 159 & 30,3 \\
\hline \multicolumn{3}{|c|}{ Tem dificuldade em pensar com clareza } \\
\hline Não & 412 & 78,5 \\
\hline Sim & 113 & 21,5 \\
\hline \multicolumn{3}{|c|}{ Tem se sentido triste ultimamente } \\
\hline Não & 315 & 60,0 \\
\hline Sim & 210 & 40,0 \\
\hline \multicolumn{3}{|c|}{ Tem chorado mais do que costume } \\
\hline Não & 415 & 79,0 \\
\hline Sim & 110 & 21,0 \\
\hline \multicolumn{3}{|c|}{ Dificuldade em encontrar satisfação nas atividades diárias } \\
\hline Não & 335 & 63,8 \\
\hline Sim & 190 & 36,2 \\
\hline \multicolumn{3}{|c|}{ Dificuldade para tomar decisões } \\
\hline Não & 390 & 74,3 \\
\hline Sim & 135 & 25,7 \\
\hline \multicolumn{3}{|c|}{$\begin{array}{l}\text { Tem dificuldade no serviço (seu trabalho é penoso, causa } \\
\text { sofrimento) }\end{array}$} \\
\hline Não & 442 & 84,2 \\
\hline Sim & 83 & 15,8 \\
\hline
\end{tabular}




\begin{tabular}{|c|c|c|}
\hline \multicolumn{3}{|c|}{ É incapaz de desempenhar um papel útil em sua vida } \\
\hline Não & 89 & 17,0 \\
\hline Sim & 436 & 83,0 \\
\hline \multicolumn{3}{|c|}{ Tem perdido o interesse pelas coisas } \\
\hline Não & 423 & 80,6 \\
\hline Sim & 102 & 19,4 \\
\hline \multicolumn{3}{|c|}{ Sente-se uma pessoa inútil, sem préstimo } \\
\hline Não & 497 & 94,7 \\
\hline Sim & 28 & 5,3 \\
\hline \multicolumn{3}{|c|}{ Tem tido a ideia de acabar com a vida } \\
\hline Não & 477 & 90,9 \\
\hline Sim & 48 & 9,1 \\
\hline \multicolumn{3}{|c|}{ Sente-se cansado o tempo todo } \\
\hline Não & 360 & 68,6 \\
\hline Sim & 165 & 31,4 \\
\hline \multicolumn{3}{|c|}{ Tem sensações desagradáveis no estomago } \\
\hline Não & 360 & 68,6 \\
\hline Sim & 165 & 31,4 \\
\hline \multicolumn{3}{|c|}{ Cansa com facilidade } \\
\hline Não & 349 & 66,5 \\
\hline Sim & 176 & 33,5 \\
\hline \multicolumn{3}{|c|}{ TMC (score do SRQ-20)* } \\
\hline Não suspeito & 330 & 62,9 \\
\hline Suspeito & 195 & 37,1 \\
\hline
\end{tabular}

Legenda: $\mathrm{N}=$ valor absoluto; $\%$ = valor percentual, $\left({ }^{*}\right)$ ponto de corte $7 / 8$.

Sobre a violência na escola, os entrevistados apontaram como principais acontecimentos a agressão verbal e/ou ameaça contra o professor $(42,9 \%)$ e a agressão física contra o professor $(22,9 \%)$, sendo o aluno apontado como o principal perpetrador em $76,8 \%$ e $80 \%$ dos casos respectivamente.

Os professores referiram também ter sofrido agressão ou ameaça com arma de fogo ou arma branca $(7,0 \%)$, ter presenciado tráfico ou venda de drogas ilícitas (10,1\%), presenciado consumo de drogas ilícitas $(11,0 \%)$ e presenciado consumo de bebida alcoólica $(4,4 \%)$ dentro da escola.

Nas quatro situações, os estudantes eram os principais perpetradores da violência $(72,8 \%, 81,1 \%, 84.5 \%$ e $78,3 \%$ respectivamente).

Analisando a associação dos TMC com as situações de violência vivenciadas pelos professores no ambiente escolar, dentre todas as variáveis questionadas apenas o consumo de bebida alcoólica dentro da escola não se mostrou associado ao desfecho pesquisado (Tabela 2).
Tabela 2 - Associação entre Transtornos Mentais Comuns e as Situações de Violência na Escola Vivenciadas pelos Professores

\begin{tabular}{|c|c|c|c|c|}
\hline \multirow[b]{2}{*}{ Variáveis } & \multicolumn{4}{|c|}{ TMC } \\
\hline & $\begin{array}{l}\text { Sim } \\
\text { n (\%) }\end{array}$ & $\begin{array}{l}\text { Não } \\
\text { n (\%) }\end{array}$ & OR & IC 95\% \\
\hline \multicolumn{5}{|c|}{ Agressão física contra o professor dentro da escola } \\
\hline Não & $281(69,4)$ & $124(30,6)$ & \multirow{2}{*}{3,28} & \multirow{2}{*}{$2,15-5,00$} \\
\hline Sim & $49(40,8)$ & $71(59,2)$ & & \\
\hline \multicolumn{5}{|c|}{$\begin{array}{l}\text { Agressão verbal e/ou ameaça contra o professor dentro da } \\
\text { escola }\end{array}$} \\
\hline Não & $220(73,3)$ & $80(26,7)$ & \multirow{2}{*}{2,87} & \multirow{2}{*}{$1,99-4,14$} \\
\hline Sim & $110(48,9)$ & $115(51,1)$ & & \\
\hline \multicolumn{5}{|c|}{$\begin{array}{l}\text { Agressão ou ameaça com arma de fogo ou arma branca den- } \\
\text { tro da escola }\end{array}$} \\
\hline Não & $319(65,4)$ & $169(34,6)$ & \multirow{2}{*}{4,46} & \multirow{2}{*}{$2,15-9,25$} \\
\hline Sim & $11(29,7)$ & $26(70,3)$ & & \\
\hline \multicolumn{5}{|c|}{ Tráfico ou venda de drogas dentro da escola } \\
\hline Não & $310(65,7)$ & $162(34,3)$ & \multirow{2}{*}{3,15} & \multirow{2}{*}{$1,75-5,67$} \\
\hline Sim & $20(37,7)$ & $33(62,3)$ & & \\
\hline \multicolumn{5}{|c|}{ Consumo de drogas dentro da escola } \\
\hline Não & $305(65,3)$ & $162(34,7)$ & \multirow{2}{*}{2,48} & \multirow{2}{*}{$1,42-4,32$} \\
\hline Sim & $25(43,1)$ & $33(56,9)$ & & \\
\hline \multicolumn{5}{|c|}{ Consumo de bebida alcoólica dentro da escola } \\
\hline Não & $319(63,5)$ & $183(36,5)$ & \multirow{2}{*}{1,90} & \multirow{2}{*}{$0,82-4,39$} \\
\hline Sim & $11(47,8)$ & $12(52,2)$ & & \\
\hline
\end{tabular}

Legenda: $\mathrm{N}$ = valor absoluto; $\%$ = valor percentual; $\mathrm{OR}=$ Odds Ratio

\section{DISCUSSÃO}

Este estudo analisou a associação entre transtornos mentais comuns (TMC) e a violência na escola. Descreve a prevalência dos TMC e das formas de violência numa realidade bastante frequente, não só da capital pernambucana, mas do Brasil: a do elevado registro de atos violentos e ameaças contra professores no ambiente escolar. O estudo assinala a importância de se conhecer como as situações no âmbito das instituições de ensino influenciam na saúde física e mental dos docentes, comprometendo a qualidade de vida daqueles que se comprometeram com a sociedade a tornar o mundo melhor através da educação.

As características gerais da população estudada como sexo, idade e escolaridade são semelhantes às encontradas em outros estudos realizados no país com professores (Souza et al., 2011; Ferreira et al., 2015).

A prevalência de casos suspeitos de TMC neste estudo foi de $37,1 \%$, valor intermediário entre os achados de Silva e Silva (2013) e Oliveira (2013), que foi de $17,8 \%$ e $54,7 \%$, respectivamente, em estudos com professores. 
Estes valores são maiores que os encontrados para a população geral pelo estudo de Coêlho et al. (2009) de $30,2 \%$ e para a população de trabalhadores urbanos pelo estudo de Farias e Araújo (2011) de 25,2\%.

Tais números sugerem que a docência, em diferentes cenários e áreas do Brasil, afeta a saúde mental do professor.

Sobre as situações de violência vivenciadas na escola, neste estudo a agressão verbal ou ameaça e a agressão física foram apontadas como as formas mais frequentes, tendo o estudante como principal perpetrador. Este resultado se assemelha ao encontrado por Tiesman, Konda, Hendricks, Mercer \& Amandus (2013) no qual $60,4 \%$ dos professores relataram ter sofrido agressão verbal e em $73 \%$ dos casos partindo dos estudantes. Kauppi \& Pörhöla (2012) avaliando a violência contra o professor a partir do bullying e da vitimização, afirmam que alguns professores atribuem a violência à falta de valorização profissional.

Costa, Barbosa e Carraro (2014) destacam que o sentimento de insegurança e fragilidade emocional que os professores enfrentam em situações de violência podem levar ao desgaste profissional, a desmotivação para o trabalho e ao adoecimento mental o que, por sua vez, poderia repercutir no absenteísmo.

$\mathrm{O}$ estudo mostra que a agressão física $(\mathrm{OR}=3,28$ IC95\%=2,15-5,00) e a agressão verbal (OR=2,87 IC95\%=1,99-4,14) contra o professor, bem como a agressão ou ameaça com arma branca ou arma de fogo $(\mathrm{OR}=4,46 \mathrm{IC} 95 \%=2,15-9,25)$, o tráfico $(\mathrm{OR}=3,15 \mathrm{IC} 95 \%$ $1,75-5,67)$ e o consumo de drogas na escola $(\mathrm{OR}=2,48$ IC95\%=1,42-4,32) estão associados aos TMC.

Sobre isso, Türküm (2011) discute que apesar de existirem normas legais para proteger os indivíduos dessas situações, muitas vezes esses não pedem ajuda e, por não obter o apoio necessário e o atendimento especializado de que necessitam para tratar o trauma causado por essa violência, acabam desenvolvendo tal tipo de transtorno.

Este estudo, por uma limitação do desenho transversal, não pode confirmar a sequência temporal dos eventos. Para aumentar a segurança dos pesquisados sobre o anonimato das informações, não foi questionado o nome das escolas nas quais os mesmos lecionavam e, por este motivo, não foi possível mapear a violência na rede pública de ensino do Município.

\section{CONSIDERAÇÕES FINAIS}

Acreditando que a escola seja um ambiente de desenvolvimento e crescimento, não somente para o aluno, mas também para o professor, é preciso uma maior compreensão sobre o fenômeno crescente da violência na escola e de como ele afeta a vida e a saúde destes trabalhadores e de todos os envolvidos. Por ser a violência um problema complexo e multifatorial, ao mesmo tempo em que se faz necessário investir na promoção da saúde mental e na capacitação do professor para o enfrentamento e prevenção da violência por meio de medidas de segurança, é preciso intervir no contexto social no qual a escola está inserida. Para tanto, é preciso dar visibilidade ao problema aumentando o debate científico e da sociedade.

\section{IMPLICAÇÕES PARA A PRÁTICA CLÍNICA}

Devido aos Transtornos Mentais Comuns serem a morbidade psiquiátrica mais prevalente na sociedade, fazse necessário a realização de estudos que investiguem a prevalência dos TMC, seus fatores associados e sua influência na saúde física e mental dos trabalhadores de modo a estimar o impacto destes diante da prática clínica. Tais investigações servirão de subsídio teórico e empírico no planejamento de ações da prática clínica, seja no âmbito da prevenção e/ou da promoção da saúde mental dos trabalhadores e/ou na reabilitação e reinserção social/profissional daqueles que necessitarem de tratamento psíquico.

\section{REFERÊNCIAS BIBLIOGRÁFICAS}

Base de Dados do Estado. (2016). Perfil municipal Jaboatão dos Guararapes. Disponível em http:// www.bde.pe.gov.br/Arquivos PerfilMunicipal/ JABOAT\%C3\%83O\%20DOS\%20GUARARAPES.pdf

Coelho F. M. C., Pinheiro R. T., Horta B. L., Magalhães P. V. S., Garcias C. M. M., e Silva C. V. (2009). Common mental disorders and chronic non-communicable diseases in adults: A population-based study. Cadernos de Saúde Pública, 25(1), 59-67. doi: 10.1590/S0102311X2009000100006

Costa M. S. G. A., Barbosa N. D., e Carraro P. R. (2014). A importância do trabalho do psicólogo escolar aos docentes em escolas públicas. Revista EIXO, 3(2), 73-80. Disponível em http://revistaeixo.ifb.edu.br/index.php/ RevistaEixo/article/viewFile/146/118 
Delcor N. S., Araújo, T. M., Reis, E. J. F. B., Porto, L. A., Carvalho, F. M., Silva, M. O., ... e Andrade, J. M. (2004). Condições de trabalho e saúde dos professores da rede particular de ensino de Vitória da Conquista, Bahia, Brasil. Cadernos de Saúde Pública, 20(1), 187-196. doi: 10.1590/S0102-311X2004000100035

Farias M. D., e Araújo T. M. (2011). Transtornos mentais comuns entre trabalhadores da zona urbana de Feira de Santana-BA. Revista Brasileira de Saúde Ocupacional, 36(123), 25-39. doi: 10.1590/S030376572011000100004

Ferreira R. C., Silveira A. P., Sá M. A. B., Feres S. B. L., Souza J. G. B., e Martins A. M. E. B. L. (2015). Transtorno mental e estressores no trabalho entre professores universitários da área da saúde. Trabalho, Educação e Saúde, 13 (Suppl. 1), 135-155.

Ferreira L. L. (2010). Relações entre o trabalho e a saúde de professores na Educação Básica no Brasil. Relatório final do Projeto "Condições de trabalho e suas repercussões na saúde dos professores de Educação Básica no Brasil”. São Paulo: Fundacentro.

Goldberg D., \& Huxley P. (1992). Common mental disorders: A bio-social model. London: Tavistock.

Instituto Nacional de Estudos e Pesquisas Educacionais Anísio Teixeira. (2015). Censo educacional 2015. Disponível em www.portal.inep.gov.br/resultados-eresumos

Kauppi T., \& Pörhöla M. (2012). School teachers bullied by their students: Teachers' attributions and how they share their experiences. Teaching and Teacher Education, 28(7), 1059-1068. doi: 10.1016/j.tate.2012.05.009

Maciel R. H., Nogueira C. V., Maciel E. C., e Aquino R. (2012). Afastamentos por transtornos mentais entre professores da rede pública do Estado do Ceará. O Público e o Privado, 19, 167-178. Disponível em http:// www.seer.uece.br/?journal=opublicoeoprivado\&pag $\mathrm{e}=$ article \&op $=$ view \&path $\% 5 \mathrm{~B} \% 5 \mathrm{D}=329 \&$ path $\% 5 \mathrm{~B} \%$ $5 \mathrm{D}=506$

Mari J. J., \& Williams P. A. (1986) A validity study of a Psychhiatric Screening Questionnaire (SRQ 20) in primary care in city of São Paulo. Brazilian Journal of Psychiatry, 148, 23-26. Disponível em https://www.ncbi. nlm.nih.gov/pubmed/3955316

Oliveira L. F. (2013). Prevalência de transtornos mentais comuns e fatores associados em professores (Dissertação de Mestrado). Universidade Federal da Bahia, Faculdade de Medicina da Bahia, Salvador/BA.
Oliveira W. C. (2012). O papel do professor diante do bullying na sala de aula (Monografia). Universidade Técnóloga Federal do Paraná, Medianeira.

Ristum, M. (2010) Violência na escola, da escola e contra a escola. In G. S. Assis, P. Constatino, e J. Q. Avanci (Orgs.), Impacto da violência na escola: Um diálogo com professores (pp. 67-93). Rio de Janeiro: Editora Fiocruz.

Santos F. P. A., Vidal L. M., Bittencourt I. S., Boery R. N. S. O., e Sena E. L. S. (2011). Estratégias de enfrentamento dos dilemas bioéticos gerados pela violência na escola. Physis: Revista de Saúde Coletiva, 21(1), 267281. doi: 10.1590/S0103-73312011000100016

Santos K. O. B., Araújo, T. M., e Oliveira, N. F. (2009). Estrutura fatorial e consistência interna do Self-Reporting Questionnaire (SRQ-20) em população urbana. Cadernos de Saúde Pública, 25(1), 214-222.

Silva L. G., e Silva M. C. (2013). Condições de trabalho e saúde de professores pré-escolares da rede pública de ensino de Pelotas, RS, Brasil. Ciência \& Saúde Coletiva, 18(11), 3137-3146.

Sousa A. J. A. (2013). Campanha de comunicação violência no trabalho (Trabalho de Projeto). Instituto Politécnico de Lisboa, Escola Superior de Comunicação Social, Lisboa. Disponível em http://repositorio.ipl. pt/bitstream/10400.21/3394/1/Viol\%C3\%AAncia\%20 no $\% 20$ Trabalho $\% 20 \% 20$ Jacinta $\% 20$ Sousa $\% 20 \% 20$ GERP\%202011-2013.pdf

Souza C. L., Carvalho F. M., Araújo, T. M., Reis E. J. F. B., Lima V. M. C., e Porto L. A. (2011). Fatores associados a patologias de pregas vocais em professores. Revista de Saúde Pública, 45(5), 914-921. doi: 10.1590/ S0034-89102011005000055

Tiesman H., Konda S., Hendricks S., Mercer D., \& Amandus H. (2013). Workplace violence among Pennsylvania education workers: Differences among occupations. Journal of Safety Research, 44, 65-71.

Türküm, A. S. (2011). Social supports preferred by the teachers when facing school violence. Children and Youth Services Review, 33(5), 644-650. Disponível em http://www.sciencedirect.com/science/article/pii/ S0190-7409(10)00349-X

World Health Organization. Division of Mental Health. (1994). A user's guide to Self-Reporting Questionnaire (SRQ). Geneva: World Health Organization. Disponível em http://apps.who.int/iris/bitstream/10665/61113/1/ WHO_MNH_PSF_94.8.pdf 